\title{
DIAGNOSTIC POSSIBILITIES AND LIMITATIONS OF URGENT INTRAOPERATIVE PATHOLOGICANATOMICAL STUDIES IN ONCOGYNECOLOGY
}

\author{
V.V. Saevets ${ }^{1,2}$, A.Y. Shamanova ${ }^{1,2}{ }^{2}$, Y.A. Semenov ${ }^{2},{ }^{3}$, A.V. Chizhovskaja ${ }^{3}$ \\ ${ }^{1}$ Chelyabinsk Regional Clinical Center of Oncology and Nuclear Medicine, Chelyabinsk, Russian Federation \\ ${ }^{2}$ South Ural State Medical University, Chelyabinsk, Russian Federation \\ ${ }^{3}$ Ministry of Health of the Chelyabinsk region, Chelyabinsk, Russian Federation
}

Introduction. One of the variants of intravital pathological and anatomical diagnostics is intraoperative pathological and anatomical ("urgent") examination, which serves for tumor process verification. Urgent intraoperative pathological and anatomical examination is widely used when there are certain differential diagnostic difficulties and limitations of instrumental methods of examination. The purpose of the study to analyze the possibilities, limitations and the accuracy of "urgent" pathologic and anatomical diagnostics in oncogynecological diseases. Materials and methods. We retrospectively analyzed medical records of 378 inpatients treated in the gynecologic oncology department of CHOKZO and NM for the period from January 2016 to January 2021. Results and discussion. Urgent histological examination was used to diagnose ovarian tumors $(82 \%, n=310)$; to evaluate the extent of a tumor process in uterine body cancer, ovarian cancer, cervical cancer (peritoneal carcinomatosis, ingrowth into adjacent tissues and organs) $(15 \%, n=57)$; less often to evaluate resection margin ablation in cervical cancer, uterine body cancer $(3 \%, n=11)$. In a comparison of conclusions after urgent morphological examination and conduction of material, discrepancies were registered only in hypodiagnostic ovarian tumors (in 5.8\% of all "urgent" ovarian tumor studies, $\mathrm{n}=18$ ), when the question about the malignancy potential of a process cannot be reliably decided. The reliability of intraoperative morphological verification of the process is $94.2 \%$. The sensitivity is $96 \%$, specificity is $97 \%$, which is consistent with the results of other studies. Conclusions. Intraoperative morphological diagnosis is a highly specific and highly sensitive method of investigation. There are limitations of this diagnostic method depending on the volume of tissue material, quality of cryostat sections, as well as tumor features (e.g., ovarian masses) consisting of heterologous morphological areas. A compliant, multidisciplinary approach is required to successfully meet the challenges of intraoperative examination.

Keywords: intraoperative morphological diagnosis, urgent histological examination, verification, surgical treatment.

\section{ВВЕДЕНИЕ}

Онкологические заболевания занимают 2 место в мире по частоте встречаемости в общей структуре, уступая место сердечно-сосудистой патологии, и являются социально-значимыми, влияющими на качество жизни и трудовой потенциал населения страны [1]. Борьба с онкопатологией, наряду с сердечно-сосудистыми и иными заболеваниями, является приоритетным направлением в Национальном проекте «Здравоохранение» Российской Федерации [2].

Онкогинекологические заболевания - это опухоли женской репродуктивной системы, к которым относятся рак тела матки, шейки матки, яичников и рак наружных половых органов (рак вульвы и влагалища). Ряд онкопатологий женской репродуктивной системы не имеет ярких клинических проявлений и не затрагивает визуальные локализации женских половых органов, что вносит некоторые затруднения для своевременной клинико-инструментальной диагностики и верификации патологического процесса на поликлиническом этапе. Это приводит к риску формирования запущенных стадий онкологического заболевания у пациентов. Нередко в ходе выбора тактики лечения распространенность патологического процесса оценивается хирургом-онкологом интраоперационно с использованием срочного интраоперационного патологоанатомического исследования (СИПИ).

Среди опухолей женской репродуктивной системы наибольшие трудности встречаются в диагностике новообразований яичников [3-5]. Инструментальные методы диагностики, ультразвуковое исследование, компьютерная и магнитно-резонансная томография имеют определенные ограничения, и окончательный диагноз может быть поставлен только после гистологической верификации опухоли. Специальным видам лечения онкопатологии всегда предшествует прижизненная морфологическая верификация процесса, которую в ряде онкогинекологических заболеваний проводят интраоперационно ввиду определенных ограничений инструментальной диагностики, а также невозможности проведения биопсии на догоспитальном этапе, например, при безуспешности верификации по биоптату кистозного образования яичника [6]. Для верификации опухолевого процесса на первом этапе, вне независимости от клинической стадии, прибегают к удалению опухоли яичника с последующим проведением срочного гистологического исследования, заключение которого будет служить основным фактором для определения объема хирургического лечения. Наиболее часто это проводится у молодых женщин, для которых вопрос о сохранении репродуктивной функции стоит остро. В этом случае по небольшому количеству материала, например, взятому в ходе резекции яичника, необходимо дать заключение о гистотипе опухоли, ее доброкачественном, пограничном или злокачественном характере. Возможны ситуации, когда хирургом проводится забор малого объема материала, например, в ходе органосохраняющих операций, или при высоком риске кровотечения от оперативного 
вмешательства (например, при диагностической лапароскопии в ходе взятия тканевого материала с очагов канцероматоза). Все это может создавать некоторые сложности в ходе патологоанатомической интраоперационной диагностики [7-10]. Опухоли яичников часто достигают больших размеров, нередко несколько десятков сантиметров, что обусловливает тяжесть состояния пациенток (от среднетяжелого до тяжелого). В таких ситуациях на СИПИ может быть прислано образование полностью. Но несмотря на наличие всего объема опухоли для диагностики, в условиях ограничения метода СИПИ, а также вероятности крайне гетерологичной морфологической структуры образования, возможны случаи гиподиагностики в ходе СИПИ.

При раке шейки матки возможно СИПИ лимфатических узлов на предмет метастатического поражения. В случаях обнаружения метастазов хирург может не проводить расширенную гистерэктомию. Учитывая особенности метастазирования, очень часто опухолевые клетки локализуются в виде очагов в синусах лимфоузла. Таким образом, репрезентативный тканевой срез лимфоузла с наличием опухолевых клеток может быть обнаружен только после проводки и серийного исследования тканевого материала.

Скудный объем материала, ограничение во времени проведения интраоперационного патологоанатомического исследования, а также невозможность исследовать в процессе СИПИ большего количества фрагментов тканевого материала, все это может вызвать сложности в постановке развернутого и подробного интраоперационного патологоанатомического заключения. При распространенных онкологических процессах в ходе оперативного вмешательства хирургу-онкологу требуется контролировать абластичность краев резекции, что также возможно с проведением СИПИ. Например, при распространенном раке шейки и тела матки оценивается линия резекции влагалища.

Ранее в онкогинекологии использовали СИПи различных показателей распространенности при раке тела матки, например, исследование глубины миометральной инвазии, а также гистотипа опухоли, что в настоящее время не имеет значения ввиду диагностики данных показателей с использованием ультразвуковых методов диагностики и прижизненной патологоанатомической диагностики соскобов эндометрия [11-13].

Таким образом, целесообразность СИПИ обоснована при дифференциально-диагностических сложностях и ограничениях инструментальных методов обследования, контроле абластичности краев резекции при распространенных опухолевых процессах. СИПИ при учете ограничения метода помогает установить гистотип опухоли, распространенность опухолевого процесса, отсутствие или наличие метастазов, что, в свою очередь, влияет на определение объема оперативного вмешательства.

Наибольшие сложности при постановке срочного интраоперационного патологоанатомиче ского заключения в онкогинекологии вызывают исследования злокачественных и пограничных опухолей, а также вторичных новообразований яичника [14-16].

По данным Волченко Н.Н. и соавт. [17], при верификациях опухолей яичников в ходе срочного интраоперационного морфологического исследо- вания чувствительность составляет 98,7\%, специфичность - 99\%. Но при этом, по мнению автора, интраоперационное заключение в предположительной форме может даваться при опухолях стромы полового тяжа и герминогенных опухолях [17].

Поиск более быстрых и доступных методов верификации диагноза (например, цитологического исследования) не всегда является информативным для диагностики рака яичников и в части случаев несет собой риск несоответствия морфологическому заключению. Так, по данным Давыдовой И. Ю. (2018) [18], при цитологическом исследовании асцитической жидкости у $60 \%$ больных найденные клетки интерпретированы как клетки рака. При гистологическом исследовании опухолевого материала данных пациенток диагноз рака был подтвержден только в 5\% случаев, у остальных 55\% больных опухоль соответствовала серозной пограничной опухоли [18]. На примере исследования 257 случаев серозной пограничной опухоли яичников, пролеченных в НМИЦ онкологии им. Н.Н. Блохина [18] за период 1970-2013 гг., авторы отмечают высокую частоту гипердиагностики; так, в 54,8\% случаев ранее установленной аденокарциномы на фоне серозной пограничной опухоли яичников после пересмотра гистопрепаратов элементов злокачественной опухоли не обнаружено. Это, в свою очередь, подтверждает объективные сложности в ходе интерпретации СИПИ опухолей яичников, когда морфологическая диагностика затруднительна из-за качества гистологической картины криостатного тканевого среза, намного уступающего таковому после плановой проводки материала, а также из-за ограничений в объеме исследуемого материала опухоли, и возможности наличия гетерологичных морфологических участков в опухолях яичников.

Метод срочного патологоанатомического исследования СИПИ давно зарекомендовал себя как эффективный и недорогостоящий. Однако перед проведением данного метода диагностики от хирурга-онколога требуется четко поставленная задача, а в зависимости от полученного гистологического заключения - правильно спланированный адекватный объем хирургического вмешательства (в соответствии с клиническими рекомендациями и стандартами оказания медицинской помощи).

Основными задачами СИПИ являются:

- морфологическая верификация диагноза при невозможности дооперационной диагностики;

- уточнение степени распространенности опухолевого процесса путем изучения зон регионарного метастазирования, близкорасположенных органов, состояния органов высокого риска метастазирования;

- установление абластичности операционных краев резекции.

В ходе срочного интраоперационного исследования от врача-патологоанатома необходимо получение быстрого и точного заключения с затратой не более 20 минут времени исследования на один тканевой образец [19]. Присланные на СИПИ фрагменты тканей замораживают в микротоме-криостате, тонкие срезы ткани окрашивают гематоксилином и эозином, исследуют методом световой микроскопии. Недостатком криостатных срезов является более низкое качество гистологических препаратов, нередко со смазанной морфологической картиной, чем при рутинном гистологическом исследовании после проводки 
тканевого материала с изготовлением парафиновых блоков. В случаях исследования опухолей, имеющих гетерологичную морфологическую картину, низкую степень дифференцировки (когда опухоли морфологически одинаковые, но гистогенетически разные), истинное заключение возможно только при исследовании всего объема опухоли с последующим проведением иммуногистохимических и молекулярно-генетических исследований [20-21] (рис. 1).
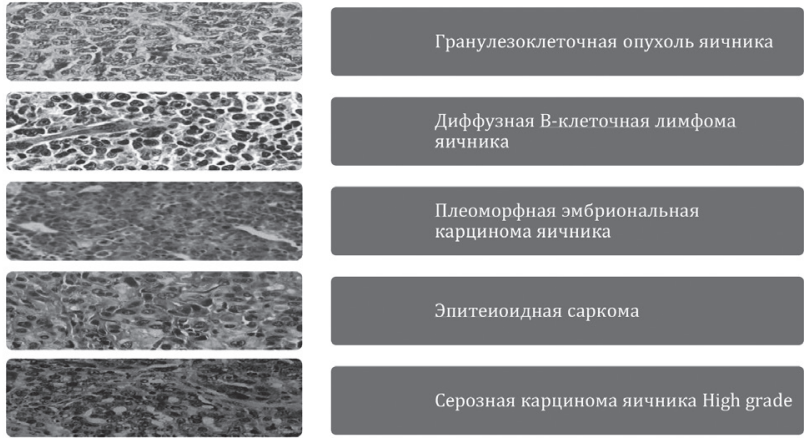

Рис. 1. Примеры морфологически сходных

но гистогенетических различных опухолей яичника (по WHO Classification of Tumours of Female Reproductive Organs. IARC: Lyon 2014)

Для качественной интраоперационной морфологической верификации диагноза необходимо:

- получение информативного операционного материала;

- правильное оформление сопроводительных документов с указанием анамнестических данных, стадии процесса, характера предоперационного лечения, сроков его проведения;

- опыт врача-патологоанатома, правильный выбор и забор наиболее репрезентативных тканевых фрагментов для СИПИ, а также подробное макро- и микроскопическое исследование операционного материала.

При объективных сложностях СИПИ возможно получение ложноположительного и ложноотрицательного заключения. Причины ложноотрицательного морфологического заключения: регрессия опухоли, вызванная неоадъювантной химиотерапией; некротические изменения ткани в ходе распада опухоли; замещение опухолевой ткани фиброзированной тканью; обильная васкуляризация опухоли; выраженная сопутствующая инфильтрация стромы новообразования гистиоцитами, лимфоцитами, плазматическими клетками, гранулоцитами, что может быть ложно трактовано как воспаление с преобладанием продуктивной тканевой реакции. Причинами ложноположительных заключений являются: резко выраженная пролиферация и реактивные изменения клеточных элементов ткани органа, выраженные воспалительные изменения с наличием крупных макрофагальных и гистиоцитарных элементов с пролиферацией эндотелия сосудов. При объективных сложностях СИПИ решение при постановке патологоанатомического заключения принимается в сторону гиподиагностики.

Цель исследования - анализ возможностей и ограничений, а также точности интраоперационной («срочной») патологоанатомической диагностики при онкогинекологических заболеваниях.

\section{МАТЕРИАЛЫ И МЕТОДЫ}

Ретроспективно проанализированы медицинские карты 378 стационарных больных и микропрепараты тканевого материала от данных пациенток, проходивших лечение в условиях онкологического гинекологического отделения ЧОКЦО и ЯМ с января 2016 года по январь 2021 года, и патологоанатомические заключения на СИПИ материала данных пациенток.

Критерии включения в исследование: все случаи онкогинекологических заболеваний за указанный период с проведением СИПИ и последующим патологоанатомическим прижизненным исследованием тканевого материала вне зависимости от локализации и характера патологического процесса. Критерии исключения: в группу исследования не включали случаи плановых хирургических вмешательств с уже верифицированными новообразованиями, а также случаи без проведения СИПИ.

Проведен анализ и сопоставление заключений срочных интраоперационных патологоанатомических исследований с клинико-инструментальными данными, а также проанализированы возможности и ограничения данного метода патологоанатомического исследования.

Для характеристики информативности метода СИПИ опухолей яичников оценивались объективные параметры: чувствительность (Se, sensitivity), специфичность (Sp, specificity). Чувствительность Se рассчитывалась по формуле:

$$
S e=(T P / T P+F N) \times 100 \%,
$$

где ТР - истинно положительные результаты;

FN - ложноотрицательные результаты.

Специфичность Sp рассчитывалась по формуле:

$S p=(T N / T N+F P) \times 100 \%$,

где TN - количество истинно отрицательных

результатов;

FP - количество ложноположительных

результатов.

За диагностический метод, используемый в качестве золотого стандарта, было принято гистологическое исследование материала в ходе плановой проводки.

\section{РЕЗУЛЬТАТЫ}

В исследовании нами установлено, что наиболее часто СИПИ применяли при диагностике опухолей яичников $(82 \% ; n=310)$; для оценки распространенности опухолевого процесса при раке тела матки, раке яичника, раке шейки матки (канцероматоз брющины, врастание в прилежащие ткани и органы) (15\%; n=57), реже - для оценки абластичности краев резекции при раке шейки матки, раке тела матки (3\%; $n=11)$ (рис. 2).

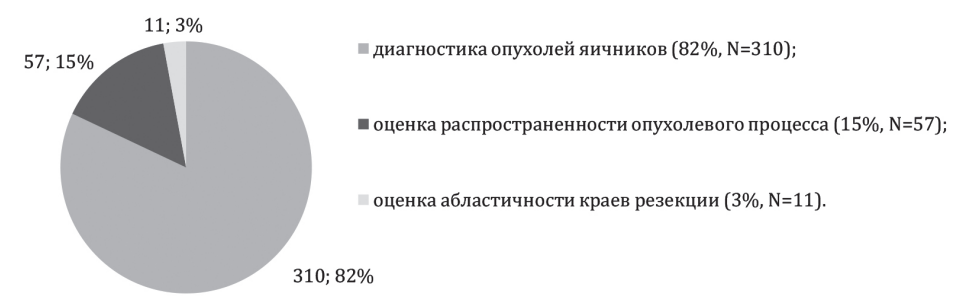

Рис. 2. Структура задач проведения СИПИ за период 2016-2021 гг. 
В ходе анализа структуры патологии опухолей яичников при СИПИ выявлено, что большую часть случаев приходится на серозную карциному $(39,4 \% ; n=122) ;$ пограничные опухоли яичников (серозные, эндометриоидные, муцинозные) $(31,2 \% ; n=97)$, в меньшей степени - на эндометриоидную карциному $(3,2 \% ; n=10)$, гранулезоклеточная опухоль и муцинозная карцинома яичника в одинаковом количестве $(2,3 \%$; $=7)$, опухоль стромы полового тяжа $(1,6 \% ; n=5)$. Доброкачественные новообразования и неопухолевая патология яичников составляют $11,3 \%$; $=35$ На вторичные 3 НО яичников (метастазы опухолей других локализаций в яичник) приходится 8,7\% (n=27) (рис. 3). В ходе СИПИ вторичных опухолей яичников $(\mathrm{n}=27)$ были верифицированы метастазы карциномы молочной железы в ткань яичника $(\mathrm{n}=14)$, перстневидноклеточной карциномы желудка $(\mathrm{n}=4)$, эндометриоидной карциномы тела матки (n=5), аденокарциномы толстой кишки $(\mathrm{n}=4)$ после планового исследования тканевого материала с применением иммуногистохимических методов исследования (ИГХ).
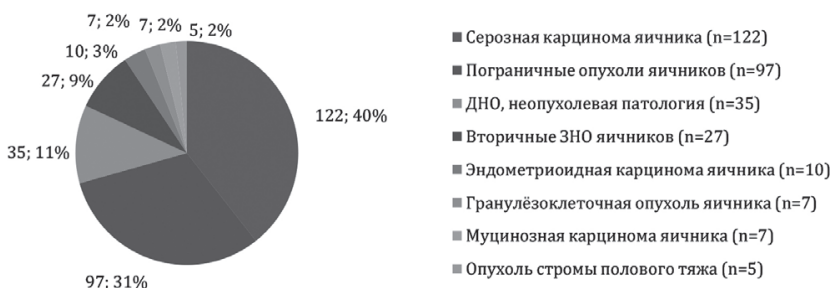

Рис. 3. Структура исследованных новообразований яичников (проведение СИПИ за период 2016-2021 гг.

с целью верификации при опухолях яичников)

В ходе сравнения заключения после СИПИ и проводки материала расхождения зарегистрированы только при диагностике опухоли яичников (в 5,8\% случаев всех СИПИ опухолей яичников; $\mathrm{n}=18$ ), причем все расхождения были связаны с первичной морфологической гиподиагностикой, когда при СИПИ верифицировались доброкачественные и пограничные опухоли яичников, а после планового исследования операционного материала яичников в большем объеме тканевых фрагментов верифицировался рак яичников. Наличие гетерологичной морфологической картины в ткани $3 \mathrm{HO}$ яичников с сочетанием участков пограничных и доброкачественных опухолей нами зарегистрировано в 75\% (n=232) всех исследованных случаев новообразований яичников (рис. 4).

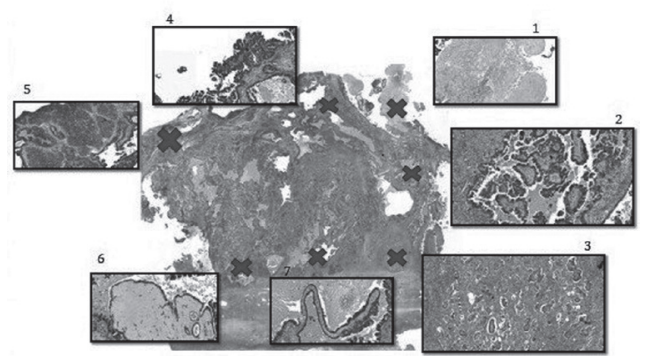

Рис. 4. Фото из архива ЧОКЦО и ЯМ. Гетерологичная морфологическая картина опухоли яичника в пределах одного тканевого фрагмента: сектор 1 - тканевой детрит; сектор 2, 4 - морфологическая картина пограничной серозной опухоли яичника; сектор 3 , 5 - морфологическая картина серозной карциномы яичника; сектор 6, 7 - гистологическая картина доброкачественной серозной опухоли яичника.

Окраска гематоксилином и эозином, ув. $\times 2$, ув. секторов 1-7 рисунка × 5
По нашим данным, в ходе сравнения заключения после срочного морфологического исследования и проводки материала расхождения в заключениях зарегистрированы только при гиподиагностике опухоли яичников (в 5,8\% случаев всех срочных исследований опухолей яичников; $\mathrm{n}=18)$, когда нельзя достоверно решить вопрос о потенциале злокачественности процесса. Также сложности диагностики могут возникать при гиперклеточности тканевых фрагментов (рис. 5).

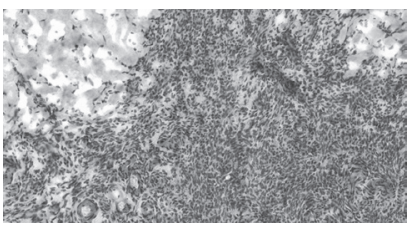

a

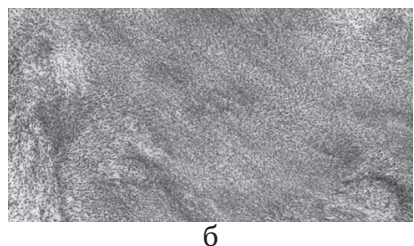

б
Рис. 5. Микрофото из архива ЧОКЦОияМ:

a - замороженный срез нормальной ткани яичника, окраска гематоксилином и эозином, ув. × 10;

6 - замороженный срез фибротекомы яичника,

окраска гематоксилином и эозином, ув. × 10

Достоверность интраоперационной морфологической верификации опухолевого процесса яичников составляет $94,2 \%$, чувствительность $96 \%$, специфичность - $97 \%$.

Сложные случаи дифференциальной диагностики первичных и вторичных опухолей яичника завершались этапом иммуногистохимического исследования (ИГХ), позволяющего определить гистогенез опухоли (рис. 6).

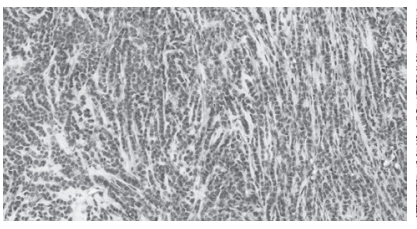
a

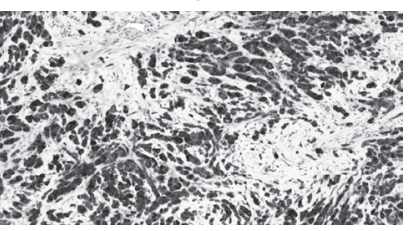

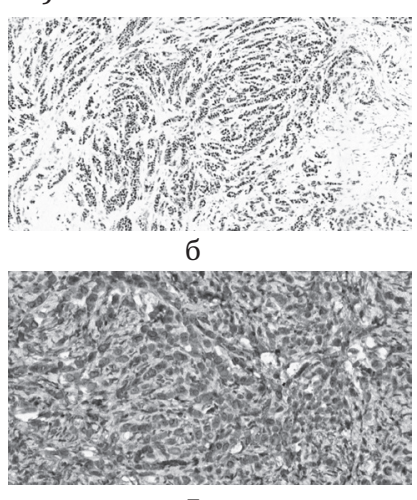

Рис. 6. Микрофото из архива ЧОКЦО и ЯМ.

Метастаз рака молочной железы в яичник. Иммуногистохимическое исследование: $\mathrm{a}-$ ткань яичника с ростом атипичных клеток средних размеров округло-овальной формы, с вакуолизированной цитоплазмой, виде коротких и длинных цепочек, окраска гематоксилином и эозином, ув. $\times 10$; б - иммуногистохимическое исследование с антителом к рецепторам Эстрогена, определяется ядерная экспрессия атипичными клетками

Estrogen Receptor; в - иммуногистохимическое исследование с антителом GCDFP, определяется экспрессия атипичными клетками GCDFP; г-

иммуногистохимическое исследование с антителом

Маммаглобин (окраска - иммуногистохимический метод, полимерная тест-система)

\section{ОБСУЖДЕНИЕ}

По данным В03, доля от всех карцином яичника при серозной карциноме low-grade составляет $5 \%$; при серозной карциноме high-grade - 70\% [20-21]. Несмотря на то, что при диагностике опухолей яичников наиболее часто верифицировались эпителиальные опухоли, проведение СИПИ также завершалось формированием заключения 
о пограничных опухолях яичников, мезенхимальных, опухолях стромы полового тяжа, вторичных новообразованиях яичников (метастазов в яичники ЗНО других локализаций) и доброкачественных опухолей яичника и неопухолевой патологией, верификация которых так или иначе меняла тактику ведения пациента. Разнообразие верифицируемых гистологических типов ЗНО яичников, а также возможность метастазирования в яичник ЗНО других локализаций (рак желудка, рак молочной железы) следует учитывать и включать в дифференциально-диагностический ряд в ходе проведения СИПИ.

Расхождения в заключениях СИПИ и после планового гистологического исследования тканевого материала (первичная морфологическая гиподиагностика) связаны, в первую очередь, с гетерологичной морфологической картиной опухоли яичников и ограниченным количеством тканевых фрагментов при СИПИ, что не позволяет исследовать полностью новообразование. Так, при исследовании муцинозных опухолей яичников (менее 10 см в максимальном размере) рекомендуется брать для гистологического исследования 1 тканевой фрагмент на каждый сантиметр образования, тогда как при опухолях более 10 см в размере по 2 тканевых фрагмента на каждый сантиметр [14].

Наши данные исследования чувствительности и специфичности метода СИПИ при опухолях яичника согласуются с результатами других российских исследователей. Так, в работе Волченко Н.Н. [15-17] показано, что сравнительно просто диагностируются неопухолевые поражения яичников, такие как фолликулярная, лютеиновая, эндометриоидная кисты, эндометриоз. Интраоперационное исследование опухолей яичников имеет ряд ограничений при пограничных опухолях. Около 20\% серозных пограничных опухолей иногда содержит небольшие очаги серозного рака, которые могут не попасть в срез при срочном исследовании из-за ограниченного количества отбора проб. По данным автора, трудности при исследовании пограничных опухолей яичников были связаны с гиподиагностикой и составили 7\% от всех случаев, что связано в основном с наличием микроочагов рака [15-17].

По нашим данным, сложности при СИПИ бывают обусловлены как особенностями самой опухоли (ее гетерогенностью, гиперклеточностью стромы с выраженной воспалительно-клеточной инфильтрацией, гистоархитектоников опухоли), так и ограничениями метода СИПИ (ограничение метода и отбора количества тканевых фрагментов). В таких ситуациях неизбежны расхождения в заключениях СИПИ и планового гистологического исследования материала с высоким риском ошибки [14]. По данным ряда авторов, чувствительность СИПИ при ЗНО яичников варьирует 86-92\%, а специфичность - 97-100\% [14-17]. Тогда как при пограничных опухолях яичников эти показатели колеблются 57-99\% для чувствительности метода и 94-98\% - для специфичности метода. В отношении доброкачественных опухолей - показатели $97-100 \%$ и 81-98\% соответственно [14-17].
Некоторыми авторами установлены основные критерии для точной цитологической диагностики опухолей яичников при экспресс-диагностике (срочной интраоперационной), в первую очередь, ЗНО. К критериям относятся высокий ядерно-цитоплазматический индекс, анизоцитоз, увеличение размеров ядра и разработан алгоритм для снижения количества расхождений с гистологическими исследованиями [22-25]. Несмотря на это, объективные трудности СИПИ сохраняются, в том числе при диагностике вторичных 3 НО яичников (метастазов опухолей иных локализаций в яичники).

Задачами ИГХ-исследования после СИПИ являлись идентификация точного гистогенеза опухоли, что имеет большое значение в определении первичного очага метастазирования, а также дифференциальная диагностика новообразований и неопухолевых процессов, доброкачественных и злокачественных опухолей, имеющих морфологическое сходство [26-32].

Вероятно, для более точного вычисления объективных параметров характеристики информативности СИПИ необходимо максимально исключить факторы, влияющие на данные показатели. Полагаем, что необходимо учитывать оптимальный диагностический золотой стандарт, а именно, гистологическое исследование материала. Для формирования группы исследования критерии включения и исключения должны быть максимально расширены с целью унификации популяции тестируемых пациентов. Ошибки в интерпретации СИПИ могут быть максимально исключены при равных условиях проведения метода (квалификационная категория специалиста-патологоанатома, опыт работы врача при СИПИ, исследование в пределах одного отделения и с использованием однотипных реагентов.

\section{ВЫВОДЫ}

Интраоперационная («срочная») морфологическая диагностика является неотъемлемой частью рутинной практики хирургов-онкологов и патологоанатомов онкологических лечебных учреждений для оказания медицинской помощи в полном объеме на этапе хирургического лечения в соответствии со стандартами и утвержденными клиническими рекомендациями. В онкогинекологии метод СИПИ играет ключевую роль при определении дальнейшей тактики хирургического лечения и влияет на объемы оперативных вмешательств.

Возможности метода СИПИ имеют ограничения из-за малых объемов исследуемой ткани, худшего качества криостатных срезов по сравнению с таковыми при плановом приготовлении микропрепаратов, ограничении во времени СИПИ, а также особенностями морфологии новообразования. СИПИ в онкогинекологии наиболее затруднительно при диагностике опухолей яичников, что неизбежно может приводить к гиподиагностике.

СИПИ показало высокую специфичность и высокую чувствительность. Для успешного решения поставленной цели и задач интраоперационного («срочного») исследования необходим комплаенсный междисциплинарный подход.

Конфликт интересов отсутствует.

\section{СПИСОК ЛИТЕРАТУРЫ}

1. Перечень социально значимых заболеваний : Постановление правительства РФ от 01.12.2004 г № 715. - Режим доступа: http://base.garant.ru/12137881/ (дата обращения: 18.04.2021). - Текст : электронный.

2. Указ Президента Российской Федерации от 07.05.2018 № 204 «0 национальных целях и стратегических задачах развития Российской Федерации на период до 2024 года». - Режим доступа: http://www.kremlin.ru/acts/bank/43027 (дата обращения: 18.04.2021). - Текст : электронный.

3. Coffey, D. Intraoperative consultation in gynecologic pathology / D. Coffey, A. L. Kaplan, I. Ramzy // Arch Pathol. - 2005. - 
№ 129. - P. 1544-1557.

4. Acs, G. Intraoperative consultation in gynecologic pathology // Semin Diagn Pathol. - 2002. - № 19. - P.237-254.

5. Boriboonhirunsarn, D. Accuracy of frozen section in the diagnosis of malignant ovarian tumor / D. Boriboonhirunsarn, A. Sermboon // J Obstet Gynaecol Res. - 2004. № 30. - P.394-399.

6. Никогосян, С. О. Современная диагностика рака яичников / С. О. Никогосян, В. В.Кузнецов // Российский онкологический журнал. - 2013. - № 5. - С. 52-56.

7. The accuracy of frozen section diagnosis of ovarian tumors / F. C. Twaalfhoven, A. A. Peters, J. B. Trimbos [et al.] // Gynecol Oncol. - 1991. - № 41. - P.189-192.

8. Accuracy of intraoperative frozen section analysis in borderline tumors of the ovary: a retrospective analysis of 96 cases and a review of the literature / Tempfer C. B., Polterauer S., Bentz E. K. [et al.]. // Gynecol Oncol. -2007.-107.- P.248-252.

9. Pre and intraoperative diagnosis of ovarian tumours: how accurate are we? / Lim F. K., Yeoh C. L., Chong S. M. [et al.] // Aust N Z J Obstet Gynaecol. - 1997. -№ 37. - P. 223-227.

10. Accuracy of frozen section (intraoperative consultation) diagnosis of ovarian tumors / Rose P. G., Nelson R. R., Hunter R. E. [et al.] // Am J Obstet Gynecol. - 1994. - Vol. 171 (3). - P.823-826.

11. Fanning, J. Intraoperative frozen section diagnosis of depth of myometrial invasion in endometrial adenocarcinoma / J. Fanning, Y. Tsukada, M. S. Piver // Gynecol Oncol. - 1990. - 37. - P.47-50.

12. Quinlivan, J. A. Accuracy of frozen section for the operative management of endometrial cancer / J. A. Quinlivan, R. W. Petersen, J. L. Nicklin // BJOG. - 2001. -108. - P.798-803.

13. Frozen section examination of endometrial curettings in the diagnosis of ectopic pregnancy / Barak S., Oettinger M., Perri A. [et al.] // Acta Obstet Gynecol Scand. - 2005. - 84. - P.43-47.

14. Опухоли яичника и маточной трубы. Морфологическая диагностика и генетика : Руководство для врачей / Ю. Ю. Андреева, Н. В. Данилова, Л. В. Москвина [и др.] ; под ред. Ю. Ю. Андреевой, Г. А. Франка. - М. : Практическая медицина, 2021. - 240 с.

15. Срочная морфологическая диагностика в онкологии / Н. Н. Волченко, О. В. Борисова, А. Г. Ермолаева [и др.] // Онкология. Журнал им. П.А. Герцена. -2015. - № 4. - С.5-11.

16. Интраоперационная срочная цитологическая диагностика в онкологии / Н. Н. Волченко, О. В. Борисова, В. Ю. Мельникова [и др.] / Новости клинической цитологии. - 2019. - Т. 23. - №2. - С.5-11.

17. Срочная морфологическая диагностика в онкологии / Н. Н. Волченко, О. В. Борисова, В. Ю. Мельникова, [и др.] / Онкология. Журнал им. П.А. Герцена. - 2020. - Т. 9. - №1. - С.5-14.

18. Давыдова, И. Ю. Серозные пограничные опухоли яичников : автореф. дис. ... д-ра мед. наук / И. Ю. Давыдова. Москва, 2018. - 43 с.

19. Приказ Министерства Здравоохранения РФ «О правилах проведения патологоанатомических исследований» от 24.03.2016 № 179н. - Режим доступа: https://normativ.kontur.ru/document?moduleId=1\&documentId=334099 (дата обращения: 18.04.2021). - Текст : электронный.

20. WHO Classification of Tumours of Female Reproductive Organs. 4th edition / R. J. Kurman, M. L. Carcangiu, C. S. Herrington, R. H. Young // IARC: Lyon. - 2014. - 262 p.

21. Ian, A. Cree. WHO Classification of Female genital tumours. 5th edition // IARC: Lyon. - 2020. - 631 p.

22. Питерская, Е. А. Диагностическая значимость цитологических признаков эпителиальных опухолей яичников / Е. А. Питерская, Г. П. Гладилин // Саратовский научно-медицинский журнал. - 2008. - № 2 (20). - С. 51-55.

23. Питерская, Е. А. Роль цитологического экспресс-метода в диагностике эпителиальных опухолей яичников / Е. А. Питерская, Г. П. Гладилин // Клиническая лабораторная диагностика. - 2008. - № 8. - С. 45.

24. Питерская, Е. А. Цитоморфологические особенности опухолей яичников / Е. А. Питерская, Г. П. Гладилин, Л. Ф. Жандарова // Фундаментальные исследования. - 2008. - № 9. - С. 101-103.

25. Питерская, Е. А. Цитологический экспресс- метод эпителиальных опухолей яичников / Е. А. Питерская, Г. П. Гладилин // Успехи современного естествознания. - 2008. - №5. - С. 88-90.

26. Петров, С. В. Руководство по иммуногистохимической диагностике опухолей человека / С. В. Петров ; под ред. С. В. Петрова, Н. Т. Райхмана. - Казань, 2000. - 288 с.

27. Автандилов, Г. Г. Медицинская морфометрия: руководство / Г. Г. Автандилов. - Москва : Медицина, 1990. - 383 с.

28. Автандилов, Г. Г. Основы патологоанатомической практики / Г. Г. Автандилов. - Москва : Медицина, 1999. - 506 с.

29. Taxy, J. B. Biopsy interpretation: the frozen section / J. B. Taxy, A. N. Husain, A. G. Montag. - 1 st ed. - 363 p.

30. Young, R. H. From Krukenberg to today: the ever present problems posed by metastatic tumors of the ovary. Part II // Adv Anat Pathol. - 2007. - № 14. - P. 149-177.

31. Lee, K. R. The distinction between primary and metastatic mucinous carcinomas of the ovary / K. R. Lee, R. H. Young // Am J Surg Pathol. - 2003. - 27. - P. 281-292.

32. Young, R. H. From Krukenberg to today: the ever present problems posed by metastatic tumors to the ovary: part 1. Historical perspective, general principles, mucinous tumors including the Krukenberg tumor // Adv Anat Pathol. - 2006. 13. - P.205-227.

\section{Сведения об авторах}

Саевец Валерия Владимировна, к.м.н. ГБУЗ "ЧОКЦО и ЯМ", г. Челябинск, Россия. ФГБОУ ВО «ЮУГМУ» Минздрава России, г. Челябинск, Россия.

ORCID: 0000-0003-2572-2408

Email: lalili2013@mail.ru

Шаманова Анна Юрьевна, к.м.н.

ГБУЗ "ЧОКЦО и ЯМ", г. Челябинск, Россия

ФГБОУ ВО «ЮУГМУ» Минздрава России,

г. Челябинск, Россия.

Email: anna-sha@bk.ru

Семенов Юрий Алексеевич, к.м.н.

Министерство здравоохранения

Челябинской области, г. Челябинск, Россия.

ФГБОУ ВО «ЮУГМУ» Минздрава России,

г. Челябинск, Россия.

Чижовская Анна Валерьевна

ГБУЗ «ОПЦ», г. Челябинск, Россия

Email: ms.chizhovskaya@mail.ru

\section{Information about the authors}

Valerija V. Saevets, PhD

Chelyabinsk Regional Clinical Center of Oncology

and Nuclear Medicine, Chelyabinsk, Russia.

South Ural State Medical University, Chelyabinsk, Russia.

ORCID: 0000-0003-2572-2408

Email: lalili2013@mail.ru

Anna Yu. Shamanova, PhD

Chelyabinsk Regional Clinical Center of Oncology

and Nuclear Medicine, Chelyabinsk, Russia.

South Ural State Medical University, Chelyabinsk, Russia.

Email: anna-sha@bk.ru

Yrii A. Semenov, PhD

Ministry of Health of the Chelyabinsk region,

Chelyabinsk, Russia.

South Ural State Medical University,

Chelyabinsk, Russia.

Anna V. Chizhovskaja

Regional Perinatal Center, Chelyabinsk, Russia

Email: ms.chizhovskaya@mail.ru 